\title{
Hodgkin lymphoma detection and survival: findings from the Haematological Malignancy Research Network
}

\section{Maxine JE Lamb, $\mathrm{PhD}^{1 *}$, Eve Roman, $\mathrm{PhD}^{2}$, Debra A Howell, $\mathrm{PhD}^{3}$, Eleanor Kane, $\mathrm{PhD}^{4}$, Timothy Bagguley, $\mathrm{MSc}^{5}$, Cathy Burton, FRCPath, MRCP6, Russell Patmore, FRCPath, MRCP7 ${ }^{7}$ Alexandra G Smith, PhD $^{8}$}

${ }^{1}$ Research Fellow, Epidemiology \& Cancer Statistics Group (ECSG), Department of Health Sciences, University of York, York, UK; ${ }^{2}$ Professor, Epidemiology \& Cancer Statistics Group (ECSG), Department of Health Sciences, University of York, York, UK; ${ }^{3}$ Senior Research Fellow, Epidemiology \& Cancer Statistics Group (ECSG), Department of Health Sciences, University of York, York, UK; ${ }^{4}$ Research Fellow, Epidemiology \& Cancer Statistics Group (ECSG), Department of Health Sciences, University of York, York, UK; ${ }^{5}$ Data Analyst, Epidemiology \& Cancer Statistics Group (ECSG), Department of Health Sciences, University of York, York, UK; ${ }^{6}$ Consultant Haematologist, Haematological Malignancy Diagnostic Service, St James's University Hospital, Leeds, UK; ${ }^{7}$ Consultant Haematologist, Queen's Centre for Oncology and Haematology, Hull and East Yorkshire Hospitals, Cottingham, UK; ${ }^{8}$ Reader, Epidemiology \& Cancer Statistics Group (ECSG), Department of Health Sciences, University of York, York, UK

\begin{abstract}
Background: Hodgkin lymphoma is usually detected in primary care with early signs and symptoms, and is highly treatable with standardised chemotherapy. However, late presentation is associated with poorer outcomes.

Aim: To investigate the relationship between markers of advanced disease, emergency admission, and survival following a diagnosis of classical Hodgkin lymphoma (CHL).

Design \& setting: The study was set within a sociodemographically representative UK populationbased patient cohort of $\sim 4$ million, within which all patients were tracked through their care pathways, and linked to national data obtained from Hospital Episode Statistics (HES) and deaths.
\end{abstract}

Method: All 971 patients with CHL newly diagnosed between 1 September 2004-31 August 2015 were followed until 18th December 2018.

Results: The median diagnostic age was 41.5 years (range $0-96$ years), $55.2 \%$ of the patients were male, $31.2 \%$ had stage IV disease, $43.0 \%$ had a moderate-high or high risk prognostic score, and $18.7 \%$ were admitted via the emergency route prior to diagnosis. The relationship between age and emergency admission was U-shaped: more likely in patients aged $<25$ years and $\geq 70$ years. Compared to patients admitted via other routes, those presenting as an emergency had more advanced disease and poorer 3-year survival (relative survival 68.4\% [95\% confidence interval $\{\mathrm{Cl}\}=60.3$ to 75.2 ] versus $89.8 \%[95 \% \mathrm{Cl}=87.0$ to 92.0$]$, respectively $[P<0.01])$. However, after adjusting for clinically important prognostic factors, no difference in survival remained.

Copyright (C) 2019, The Authors;

DOI:10.3399/

bjgpopen $19 \times 101668$ 
Conclusion: These findings suggest that $\mathrm{CHL}$ survival as a whole could be increased by around $4 \%$ if the cancer in patients who presented as an emergency had been detected at the same point as in other patients.

\section{How this fits in}

In order to improve survival, the NHS Long Term Plan aims to increase the number of cancers diagnosed at stage I-II from $50 \%$ to $75 \%$.

In $\mathrm{CHL}$, a highly treatable haematological malignancy, emergency admission prior to diagnosis was used as a proxy for delayed diagnosis. It was found that younger and older patients, patients with comorbidities, and those with advanced disease were more likely to experience delay.

As expected, survival was poorer in this group, but importantly, after adjusting for prognostic factors, no differences in outcome were seen; indicating that if the cancer in patients presenting via an emergency route was diagnosed at the same point as patients presenting via other routes, outcomes would be equal.

These findings support the new NHS initiative, but it remains to be seen whether the targets are achievable in cancers with symptoms that are often vague, intermittent, and slow to progress.

\section{Introduction}

The relationship between late diagnosis and poor outcome is recognised for many cancers, with delay often resulting in more advanced disease, worse survival, increased risk of complications, and impaired quality of life. ${ }^{1-3}$ Hence, over recent decades the UK Department of Health has introduced a series of interventions aimed at facilitating earlier diagnosis. In primary care settings, this includes referral guidance to aid GP identification of cancer symptoms and the introduction of suspected cancer pathways to minimise time to specialist secondary care consultation. ${ }^{4}$ The latter includes 'urgent' referral pathways (consultation with a hospital specialist within 2 weeks, known as the '2week wait') and 'very urgent' (within 48 hours) for children and young adults. ${ }^{4}$ More recently, the NHS Long Term Plan ${ }^{5}$ introduced further important targets aimed at improving cancer outcomes, including an increase in the proportion of cancers identified at an early stage, from around one-half at present to three-quarters by 2028.

Hodgkin lymphoma has two distinct subtypes: $\mathrm{CHL}$ and nodular lymphocyte predominant Hodgkin lymphoma (NLPHL). The present report is restricted to $\mathrm{CHL}$, which accounts for around $90 \%$ of all newly diagnosed Hodgkin lymphomas and has a characteristic bimodal age distribution with a peak in younger adults and a further peak in older adults. ${ }^{6} \mathrm{CHL}$ can be further subdivided into nodular sclerosing, mixed cellularity, lymphocyte rich, and lymphocyte depleted Hodgkin lymphoma. Timely diagnosis is of particular interest in the context of $\mathrm{CHL}$, since it typically presents with early signs and symptoms and is highly treatable with standardised chemotherapy; the 5-year survival is around $85 \%,{ }^{7-9}$ decreasing to around $70 \%$ in those diagnosed at an advanced stage. ${ }^{7}$ By contrast, the clinical course of the rarer NLPHL is generally indolent, immediate treatment is not always required, and relative survival approaches that of the general population. ${ }^{9-12}$

$\mathrm{CHL}$ presents several challenges to detection in primary care: it is comparatively rare, accounting for $<1 \%$ of all cancers diagnosed each year in the $\mathrm{UK}^{8}$ and can be diagnosed at any age. ${ }^{7}$ Furthermore, while patients may present with classic symptoms including neck lumps, itching, and B symptoms (such as night sweats, fever, and weight loss), these, along with other symptoms, can be intermittent and slow to progress, as well as being indicative of more commonly occurring conditions. ${ }^{9,13,14}$

However, information on the relationship between presentation mode, disease stage, and survival for $\mathrm{CHL}$ is lacking. With the aim of providing contemporary population-based evidence to address this gap, clinical data and HES from an established UK patient cohort ${ }^{15}$ were used to explore the relationship between markers of diagnostic delay and survival in patients with newly diagnosed CHL. 


\section{Method}

Data are from the UK's Haematological Malignancy Research Network (HMRN; www.hmrn.org) which, with a catchment population of around 4 million that is sociodemographically representative of the UK as a whole, generates 'real-world' data that can be extrapolated to the general patient population (adults and children). Full details of HMRN's methods and ethical approvals have been published elsewhere. ${ }^{15,16}$ Briefly, initiated in September 2004, HMRN operates on a legal basis that permits all diagnostic, prognostic, treatment, and outcome data to be collected from clinical records, as well as linkage to nationwide information on deaths, cancer registrations, and HES without explicit consent. All diagnoses across HMRN's 14 hospitals ( 2400 per year) are made and coded to the latest World Health Organization International Classification of Diseases for Oncology (WHO ICD-O3) by specialist haematopathologists at a single fully-integrated laboratory, the Haematological Malignancy Diagnostic Service (www.hmds.info).

The present report includes data on 971 patients newly diagnosed with CHL between 1 September 2004 and 31 August 2015, all of whom were followed-up until 18 December 2018. Disease stage was based on the modified Ann Arbor classification (I-IV), ${ }^{17}$ performance status was assessed using the Eastern Oncology Cooperative Group's (ECOG) scale (ranging from 0 'able to carry out normal activity without restriction' to 4 'completely disabled; cannot carry out any self-care'), ${ }^{18}$ and the Hodgkin lymphoma-specific International Prognostic Score ([IPS] Hasenclever Index, developed for use in patients with advanced disease) was calculated for all patients. ${ }^{19}$ Patients were classified as treated with curative intent if they received intensive chemotherapy, as per national guidelines. ${ }^{6}$ Presence or absence of disease-associated systemic symptoms (B symptoms) was also recorded. Additional information on preceding comorbidities was obtained from inpatient HES using validated ICD10 codes for the 17 conditions in the Charlson Comorbidity Index. ${ }^{20-23}$ Pre-diagnostic emergency admissions were identified in HES using 'Routes-to-Diagnosis' methods, which include: an emergency inpatient admission originating via Accident \& Emergency attendance, GP, Bed Bureau transfer, or consultant outpatient clinic within 28 days of diagnosis. ${ }^{24-26}$ The income domain of the Index of Multiple Deprivation (IMD), grouped into quintiles (with Q1 representing the most affluent fifth of the population), was used as a marker of socioeconomic status. ${ }^{27}$

\section{Statistical analysis}

Analyses were conducted using Stata (version 15.1) and R (version 3.2.2). Logistic regression was used to calculate odds ratios (ORs). Cox proportional hazards regression and Stata's 'strel' program,

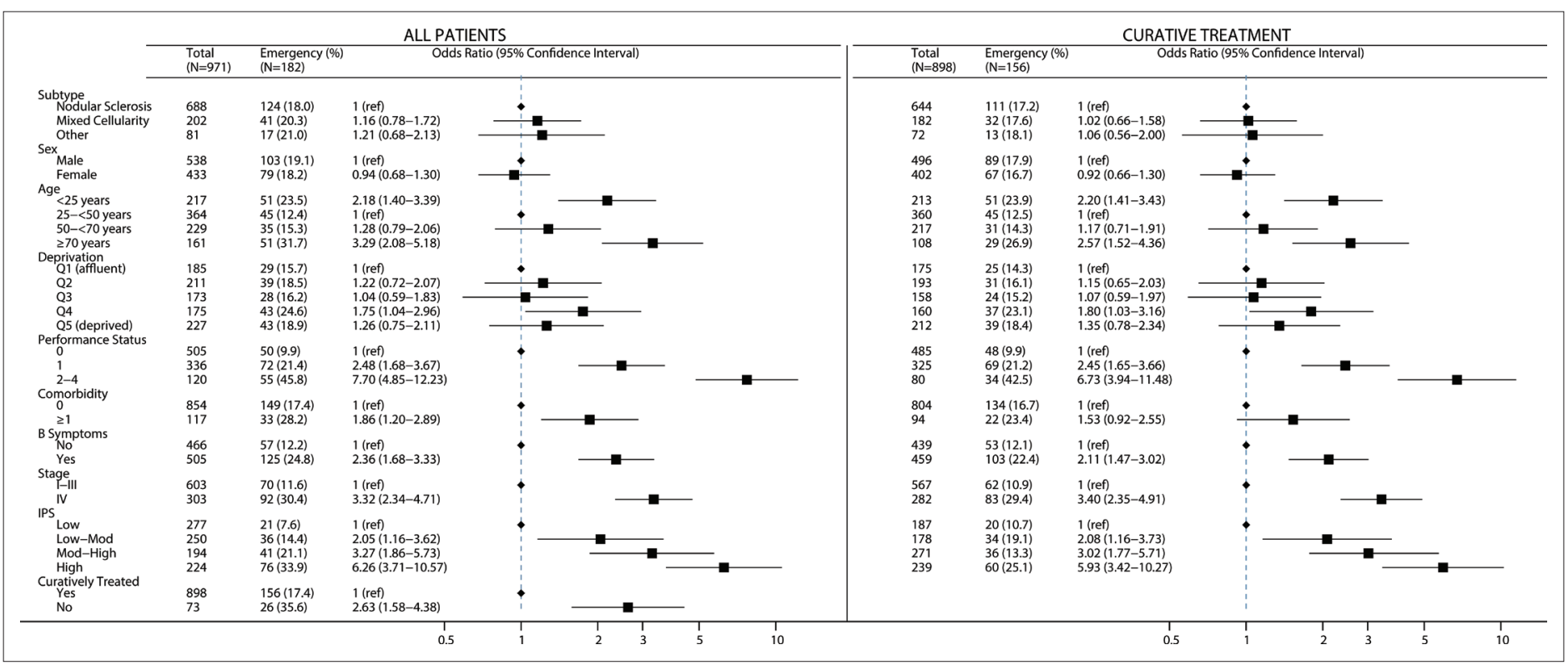

Figure 1 Patients with classical Hodgkin lymphoma distributed by demographic and clinical prognostic characteristics, with ORs (95\% Cls) for diagnosis following emergency admission presented for all patients and those treated with curative intent (data from HMRN diagnoses 2004-2015). IPS = International Prognostic Score 
which is based on the maximum likelihood method for individual records, ${ }^{28}$ were used to calculate overall survival and relative survival, respectively. The latter is a standard approach commonly used in population-based studies using national life tables ${ }^{29}$ to take into account other causes of death. Adjusted survival curves were produced using the average approach. Firstly, propensity scores were calculated, based on the probability of emergency admission predicted using logistic regression adjusted for ECOG status, B symptoms, disease stage, and other specific components of the IPS (such as albumin, haemoglobin, and lymphocyte count). These probability predictions were then scaled to the proportion of patients in each group to give the adjusted curve weights. Cox proportional hazards regression survival curves were then estimated for all combinations of the covariates included in the propensity scores plus age and curative treatment, and a weighted mean of the curves was calculated to adjust for the mix among patients, with emergency admission compared to all other routes. ${ }^{30}$

\section{Results}

The median diagnostic age of the 971 patients with $\mathrm{CHL}$ was 41.5 years and $55.2 \%$ were male. Nodular sclerosis was the most common subtype ( $n=688,70.9 \%$, median age 36.0 years), followed by mixed cellularity ( $n=202,20.8 \%, 59.6$ years), and lymphocyte rich ( $n=26,2.7 \%, 51.6$ years). CHL subtype was not characterised for the remaining patients ( $n=55,5.7 \%, 53.6$ years). In total, 182 (18.7\%) were diagnosed following emergency admission; 303 patients (31.2\%) had stage IV disease at diagnosis; $418(43.0 \%)$ had a moderate-high or high-risk IPS; and 898 (92.5\%) were treated with curative intent (Figure 1).

The relationship between emergency admission and age was U-shaped, with patients aged $<25$ years or $\geq 70$ years the most likely to present via this route (Figure 1, Supplementary Table 1): the respective ORs were $2.18(95 \% \mathrm{Cl}=1.40$ to 3.39$)$ and $3.29(95 \% \mathrm{Cl}=2.08$ to 5.18$)$. Patients with an emergency admission tended to have higher stage disease (OR $3.32,95 \% \mathrm{Cl}=2.34$ to 4.71 ); more comorbidities (OR 1.86; $95 \% \mathrm{Cl}=1.20$ to 2.89 ); at least one $\mathrm{B}$ symptom (OR $2.36,95 \% \mathrm{Cl}=$ 1.68 to 3.33 ); and poorer performance status (OR 7.70, $95 \% \mathrm{Cl}=4.85$ to 12.23 ). No differences were detected by $\mathrm{CHL}$ subtype, sex, or socioeconomic status. The 73 patients who were not treated with curative intent were also more likely to have had an emergency admission (OR $2.63,95 \% \mathrm{Cl}=1.58$ to 4.38); but their exclusion had no marked effect on the relationship between emergency admission and patient demographic and prognostic characteristics (Figure 1).

Three-year relative survival estimates for all patients and those treated curatively were $86.7 \%$ $(95 \% \mathrm{Cl}=84.0$ to 89.0$)$ and $90.1 \%(95 \% \mathrm{Cl}=87.5$ to 92.1$)$, respectively (Supplementary Table 2$)$. As expected, given the strength of the associations seen in Figure 1, outcomes were significantly poorer for patients with an emergency admission compared to those diagnosed via other routes: the 3 -year relative survival estimates were $68.4 \%(95 \% \mathrm{Cl}=60.3$ to 75.2$)$ and $89.8 \%(95 \% \mathrm{Cl}=$ 87.0 to 92.0), respectively. Figure 2 shows overall and relative survival for emergency compared to non-emergency admissions by age strata, for all patients $(A 1-4)$ and those treated with curative intent (B1-4). In patients aged $\leq 25$ years (Figure 2: A1, B1), no survival differences are evident between those with and without emergency admission. However, at older ages diagnosis following an emergency admission was associated with significantly poorer survival than diagnosis following other routes: respective 3-year relative survival estimates for emergency and non-emergency admissions were $55.4 \%(95 \% \mathrm{Cl}=36.8$ to 70.5$)$ and $86.6 \%(95 \% \mathrm{Cl}=80.8$ to 90.1$)$ in patients $50-70$ years, and $23.4 \%(95 \% \mathrm{Cl}=12.4$ to 36.3$)$ and $50.4 \%(95 \% \mathrm{Cl}=39.5$ to 60.3$)$ in patients $\geq 70$ years of age (Figure 2: A3, A4).

For emergency admissions, mortality was particularly high immediately after diagnosis (Figure 3: A1), although the disparity was marginally less marked when analyses were restricted to patients treated with curative intent (Figure 3: B1). However, for patients with an emergency admission surviving 1-year (conditional survival) mortality did not return to the background rate, with a 3-year relative survival of $88.7 \%(95 \% \mathrm{Cl}=81.0$ to 93.3$)$ compared to $96.1 \%(95 \% \mathrm{Cl}=94.0$ to 97.4$)$ in those without an emergency admission. Overall survival estimates at 1, 3, and 5 years, stratified by prognostic factors and emergency admission, are included in Supplementary Table 3. Importantly, when the prognostic profile of patients with emergency admission was adjusted to mirror that of other routes, the differences between groups disappeared (Figure 3: A2 and B2). The factor most influential in these adjustments was performance status, while albumin and haemoglobin also had an impact (Supplementary Figure 1). In practice, this implies that if patients who presented by the 


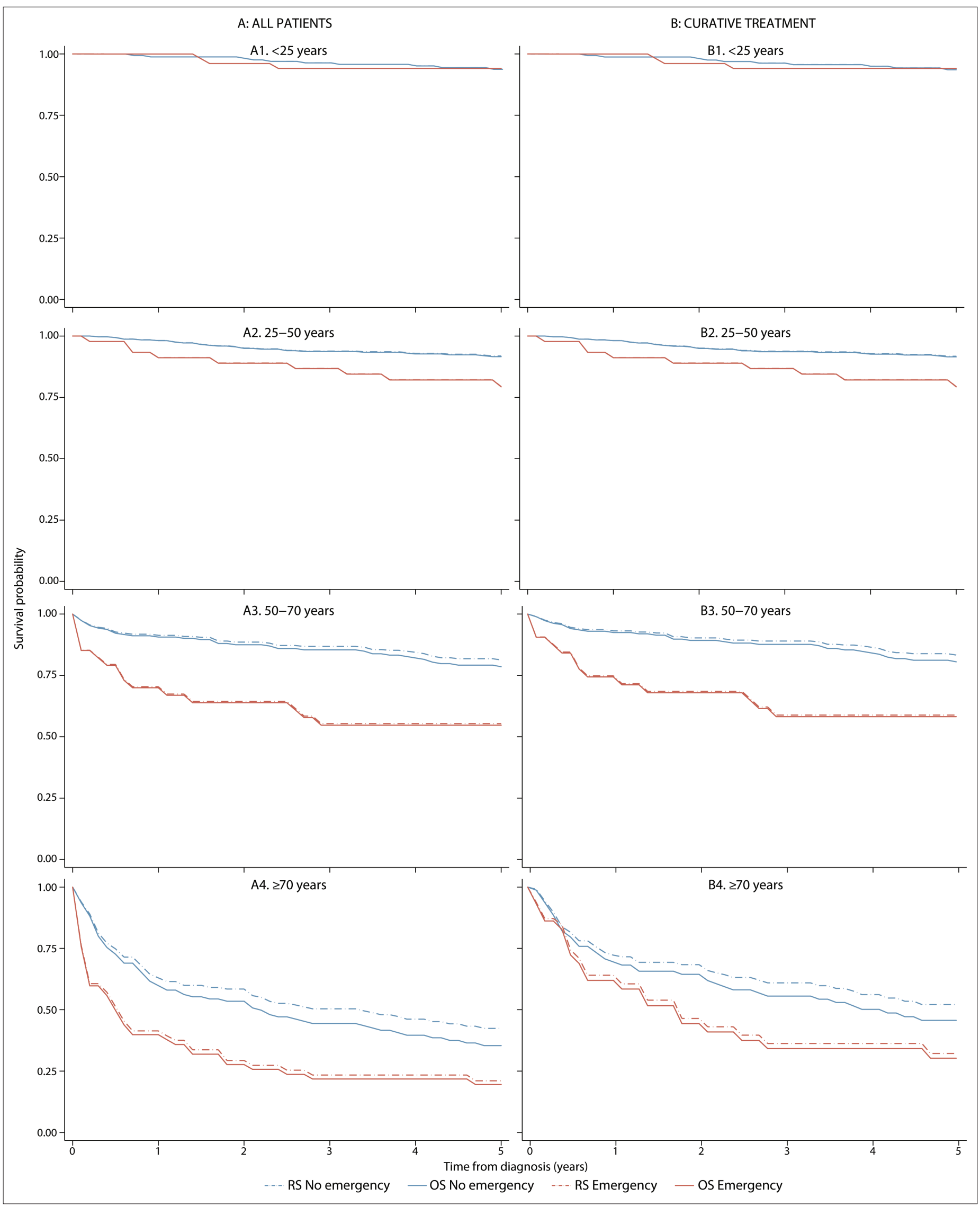

Figure 2 Classical Hodgkin lymphoma overall survival (OS) and relative survival (RS) curves for all patients (A) and those treated with curative intent (B), stratified by emergency admission status and age (data from Haematological Malignancy Research Network diagnoses 2004-2015) 


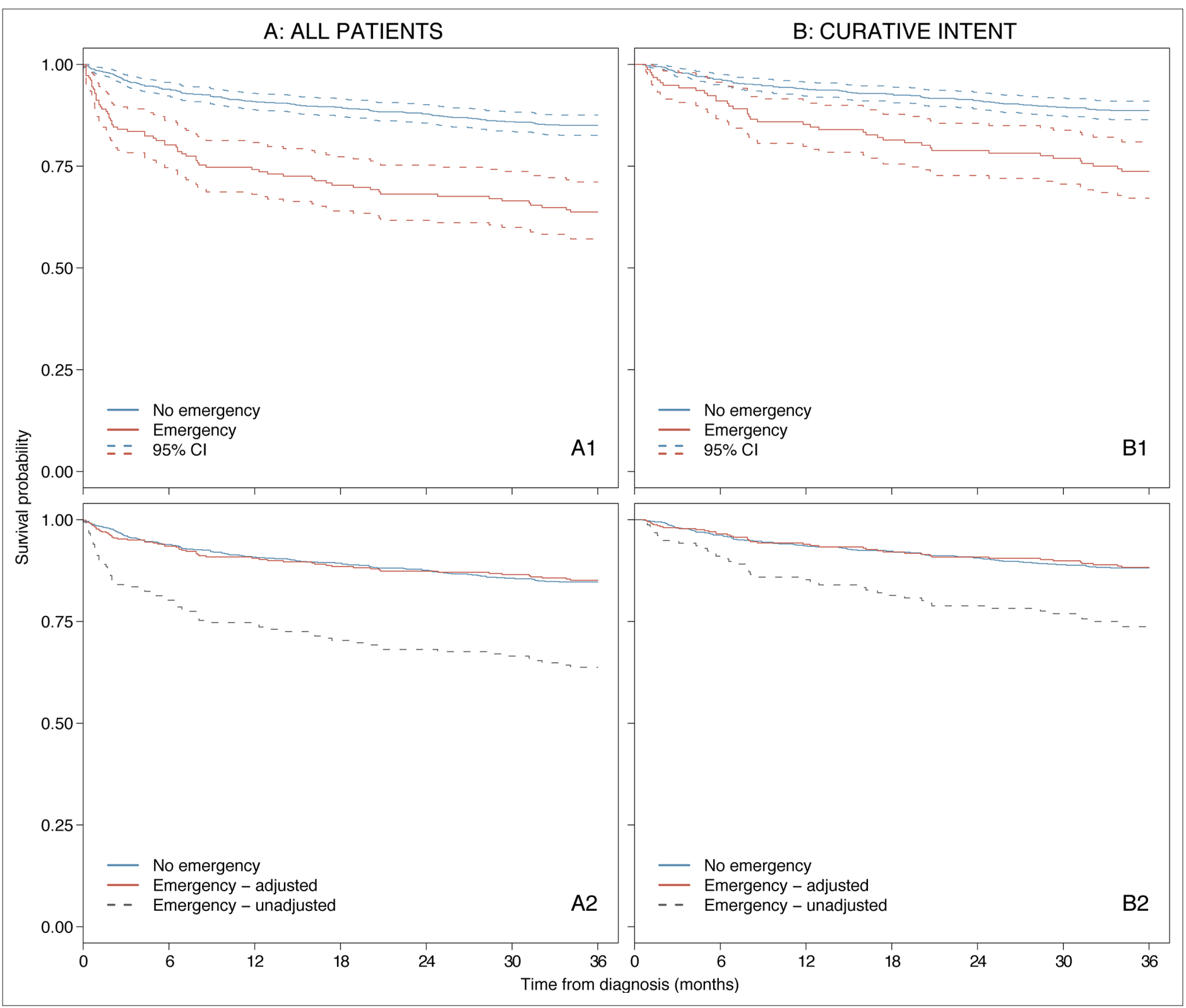

Figure 3 Classical Hodgkin lymphoma survival curves for all patients (A) and those treated with curative intent (B). Overall survival in non-emergency versus emergency admission with 95\% Cls (A1, B1) and non-emergency versus emergency adjusted for prognostic factors (A2, B2). ${ }^{a}$ (data from $\mathrm{HMRN}$ diagnoses 2004-2015)

aSurvival adjusted for age, performance status (ECOG), B symptoms (yes/no), disease stage, specific components of IPS (albumin, haemoglobin, and lymphocyte count), and treatment with curative intent (yes/no) (A2 only). Survival curves are weighted so that the patient mix with respect to ECOG status, B symptoms, disease stage, and specific components of IPS among those presenting as an emergency is similar to those who presented via other routes.

ECOG = Eastern Oncology Cooperative Group. IPS = International Prognostic Score

emergency route were diagnosed at the same point in their disease course as those who did not, the 3-year overall survival in the whole study population would equal that in the group who did not have an emergency admission; increasing from $81.1 \%(95 \% \mathrm{Cl}=78.5$ to 83.5$)$ to $85.2 \%(95 \% \mathrm{Cl}=$ 82.5 to 87.5 ); a difference of around $4 \%$ (Supplementary Table 3). 


\section{Discussion}

\section{Summary}

Linking contemporary high-quality clinical information with data contained in NHS administrative databases, this is the first population-based study to examine CHL-specific evidence about the impact of emergency admission and disease stage on survival. This study clearly identifies the substantial benefits to be gained from early $\mathrm{CHL}$ diagnosis, principally among older adults. Within this large UK population-based cohort (diagnoses 2004-2015, followed-up to December 2018), almost one in five patients were diagnosed following an emergency hospital admission, and these were more likely be diagnosed with stage IV disease. This route to diagnosis was associated with significantly worse survival than all other modes of presentation, with prognostic data indicating that the difference was largely driven by more advanced disease. Indeed, the striking disparity, seen for all patients and those treated with curative intent, disappeared when standard prognostic factors were adjusted for; raising the possibility that $\mathrm{CHL}$ survival as a whole could be increased by around $4 \%$ if the cancer in patients who presented as an emergency had been detected at the same point as in other patients. A potentially modifiable difference of this magnitude is broadly similar to that seen in successful treatment trials. ${ }^{31,32}$

\section{Strengths and limitations}

This study's findings can be extrapolated to the national population because the sociodemographic profile of HMRN's catchment population, which at around 4 million accounts for $6 \%$ of the UK's estimated total, is broadly representative of the UK as a whole and clinical practice adheres to national guidelines. ${ }^{15}$ Critically, the fact that diagnoses within HMRN are made and coded by clinical experts, also enabled the distinction between CHL and NLPHL, which are often combined in population-based studies despite substantial differences in presentation, management, and outcome. ${ }^{10,11,33}$ Moreover, in addition to this, it was also possible to differentiate between $\mathrm{CHL}$ subtypes; patients with mixed cellularity CHL had poorer 3-year relative survival than those with nodular sclerosing CHL: 79.5\% (95\% $\mathrm{Cl}=72.0$ to 85.1$)$ and $89.4 \%(95 \% \mathrm{Cl}=86.4$ to 91.8$)$ respectively (Supplementary Table 2), and the impact of emergency admission on relative survival was greatest in patients with mixed cellularity CHL (37\% difference versus 15\% difference: Supplementary Table 2). However, as mixed cellularity CHL is more common in older patients and nodular sclerosing $\mathrm{CHL}$ in younger patients (median age 59.6 years and 36.0 years respectively), the difference in outcomes is likely due, at least in part, to age. Importantly, in contrast to clinical trials, selection bias is not an issue within HMRN as all diagnoses are automatically included; and the availability of detailed clinical data enables potentially confounding prognostic factors to be considered in the analyses.

With respect to the use of HES, the authors previously demonstrated an association between emergency admission and survival in aggressive non-Hodgkin lymphoma that, in contrast to the findings for $\mathrm{CHL}$ presented here, remained after adjusting for prognostic and demographic factors. ${ }^{34}$ Undoubtedly, however, lack of primary care data limits the analysis that can be performed. A study by Abel and colleagues reported that $11.9 \%$ of patients with Hodgkin lymphoma (all subtypes combined) who presented via an emergency route and participated in the 2010 English Cancer Patient Experience Survey, had not visited their GP prior to admission. ${ }^{35}$ Unfortunately, it was not possible to investigate patterns and referrals from primary care, as national linkage to these data is currently not possible. Furthermore, as Hodgkin lymphoma is a rare disease and despite the inclusion of 971 cases in this population-based study, after stratification by emergency admission the number of subjects in some categories is small, making comparisons difficult.

\section{Comparison with existing literature}

This study's findings are broadly similar to those published by the National Cancer Registration and Service (NCRAS) in terms of overall survival for all Hodgkin lymphoma subtypes combined, and emergency admission frequency. ${ }^{36}$ Relative survival estimates are also comparable to those published by the US's Surveillance, Epidemiology and End Results programme. ${ }^{37}$ However, the general absence of clinical data on prognostic factors and treatment, mean that more granular comparisons in these registries are not possible. However, analyses of NCRAS data for several common cancers (colorectal, cervical, breast, lung, and prostate) reported that older age and advanced stage disease at diagnosis 
was predictive of emergency admission. ${ }^{38}$ In these cancers, after adjustment for age, stage, and comorbidity, emergency admission was still associated with poorer 1 -year survival. ${ }^{38}$ However, they could not adjust for performance status, which in this study's data has the strongest influence on survival.

The relationship between age at diagnosis and odds of having an emergency admission prior to diagnosis was U-shaped in the current data. The higher proportion of emergency admissions in younger patients may be a result of the 48-hour referral recommended in children and young adults suspected to have cancer, ${ }^{4}$ and the increased presence of comorbidities in older patients may impact on the higher odds of emergency admission. ${ }^{39}$ Importantly, no difference in outcomes among younger ( $<25$ years) patients with and without an emergency admission was seen. This was in contrast to older adults ( $\geq 70$ years) whose survival was poorer if diagnosed following an emergency admission, an association that remained in those treated curatively. However, it is well recognised that despite $\mathrm{CHL}$ being curable, outcomes in older patients are poor, which is generally attributed to lower tolerance of standardised chemotherapy regimens, increased comorbidities, and poorer performance status. ${ }^{6,40,41}$

\section{Implications for research and practice}

The vast majority of patients seek GP help for their symptoms at some time prior to cancer identification, including those who later go on to have a pre-diagnostic emergency admission. ${ }^{35,42}$ The opportunity to identify potential symptoms and refer patients earlier for specialist hospital consultation cannot therefore be ignored. Presently, however, raising suspicion of $\mathrm{CHL}$, and indeed other haematological malignancies in primary care, is considered difficult, ${ }^{43,44}$ not least because of the absence of clear symptoms, specific diagnostic blood tests, and realistic screening programmes, which mean repeat appraisal, help-seeking, and hospital referral is often required prior to diagnosis. ${ }^{13,45}$ Analyses of time trends of English data have shown an overall decrease in diagnoses following emergency presentation of $3 \%$ over the period 2006-2013; however, the reduction seen for Hodgkin lymphoma was only $1 \% .{ }^{46}$ Although the means of resolving such issues are far from obvious, research to further understanding of patient-healthcare professional interactions would inform the development of initiatives to promote early diagnosis.

This study's findings demonstrate the importance of existing national strategies including: safety netting in primary care, with timely post-investigation review as well as scheduled and/or patientinitiated follow-up; Rapid Diagnostic Centres for use when presenting symptoms are vague and do not meet urgent referral criteria; and faster diagnosis standard. ${ }^{4,5}$ Specific to Hodgkin lymphoma, urgent referral and investigation of older patients has been suggested for unexplained lymphadenopathy. ${ }^{14}$ The effectiveness of such measures in increasing the proportion of early stage cancer diagnoses, as described in the NHS Long Term Plan, ${ }^{5}$ remains to be seen.

In conclusion, one-fifth of patients were diagnosed with $\mathrm{CHL}$ following an emergency admission. These patients had more advanced disease and had poorer survival. As the difference in survival did not remain after adjustment for prognostic factors, the findings suggest that if $\mathrm{CHL}$ had been detected at the same point as in patients presenting via other routes, the 3 -year survival from $\mathrm{CHL}$ in the whole study population could be increased by approximately $4 \%$.

Funding

HMRN is funded by Bloodwise (grant number 15037) and receives support from NHS clinical and administrative staff across the study area.

Ethical approval

HMRN has ethical approval (refernce number: REC 04/01205/69) from Leeds West Research Ethics Committee, R\&D approval from each Trust, and exemption from Section 251 (formally Section 60) of the Health \& Social Care Act (2001) (PIAG 1-05(h)/2007).

Provenance

Freely submitted; externally peer reviewed 


\section{References}

1. Thomson CS, Forman D. Cancer survival in England and the influence of early diagnosis: what can we learn from recent EUROCARE results? Br J Cancer 2009; 101(S2): S102-S109. DOI: https://doi.org/10.1038/sj.bjc.6605399

2. Howell D, Smith A, Appleton $S$, et al. Multiple myeloma: routes to diagnosis, clinical characteristics and survival findings from a UK population-based study. Br J Haematol 2017; 177(1): 67-71. DOI: https://doi.org/10.1111/bjh. 14513

3. Zhou Y, Abel GA, Hamilton W, et al. Diagnosis of cancer as an emergency: a critical review of current evidence. Nat Rev Clin Oncol 2017; 14(1): 45-56. DOI: https://doi.org/10.1038/nrclinonc.2016.155

4. National Institute for Health and Care Excellence. Suspected cancer: recognition and referral. 2015; https://www. nice.org.uk/guidance/ng12/resources/suspected-cancer-recognition-and-referral-1837268071621 (accessed 20 Nov 2019).

5. NHS England. NHS Long Term Plan. 2019; https://www.longtermplan.nhs.uk/publication/nhs-long-term-plan/ (accessed 20 Nov 2019).

6. Follows GA, Ardeshna KM, Barrington SF, et al. Guidelines for the first line management of classical Hodgkin lymphoma. Br J Haematol 2014; 166(1): 34-49. DOI: https://doi.org/10.1111/bjh.12878

7. Howlader N, Noone A, Krapcho M. SEER Cancer Statistics Review, 1975-2016. 2018; https://seer.cancer.gov/csr/ 1975_2016/ (accessed 26 Nov 2019).

8. Cancer Research UK. Hodgkin lymphoma statistics; https://www.cancerresearchuk.org/health-professional/cancerstatistics/statistics-by-cancer-type/hodgkin-lymphoma/ (accessed 20 Nov 2019).

9. Smith A, Crouch S, Lax S, et al. Lymphoma incidence, survival and prevalence 2004-2014: sub-type analyses from the UK's haematological malignancy research network. Br J Cancer 2015; 112(9): 1575-1584. DOI: https://doi.org/ 10.1038/bjc.2015.94

10. Advani RH, Hoppe RT. XVIII. Management of nodular lymphocyte predominant Hodgkin lymphoma. Hematol Oncol 2015; 33(Suppl 1): 90-95. DOI: https://doi.org/10.1002/hon.2226

11. McKay $P$, Fielding $P$, Gallop-Evans $E$, et al. Guidelines for the investigation and management of nodular lymphocyte predominant Hodgkin lymphoma. Br J Haematol 2016; 172(1): 32-43. DOI: https://doi.org/10.1111/ bjh.13842

12. Wilson MR, Bagguley $T$, Smith $A$, et al. Frontline management of nodular lymphocyte predominant Hodgkin lymphoma - a retrospective UK multicentre study. Br J Haematol 2019; 186(6). DOI: https://doi.org/10.1111/bjh. 16109

13. Howell DA, Hart RI, Smith AG, et al. Disease-related factors affecting timely lymphoma diagnosis: a qualitative study exploring patient experiences. Br J Gen Pract 2019; 69(679): e134-e145. DOI: https://doi.org/10.3399/ bjgp19X701009

14. Shephard EA, Neal RD, Rose PW, et al. Quantifying the risk of Hodgkin lymphoma in symptomatic primary care patients aged $\geq 40$ years: a case-control study using electronic records. Br J Gen Pract 2015; 65(634): e289-e294. DOI: https://doi.org/10.3399/bjgp15X684805

15. Smith A, Howell D, Crouch S, et al. Cohort profile: the Haematological Malignancy Research Network (HMRN): a UK population-based patient cohort. Int J Epidemiol 2018; 47(3): 700-700g. DOI: https://doi.org/10.1093/ije/ dyy044

16. Smith A, Roman E, Howell D, et al. The haematological malignancy research network (HMRN): a new information strategy for population based epidemiology and health service research. Br J Haematol 2010; 148(5): 739-753. DOI: https://doi.org/10.1111/j.1365-2141.2009.08010.x

17. Lister TA, Crowther D, Sutcliffe SB, et al. Report of a committee convened to discuss the evaluation and staging of patients with Hodgkin's disease: Cotswolds meeting. JCO 1989; 7(11): 1630-1636. DOI: https://doi.org/10.1200/ JCO.1989.7.11.1630

18. Oken MM, Creech RH, Tormey DC, et al. Toxicity and response criteria of the Eastern Cooperative Oncology Group. Am J Clin Oncol 1982; 5(6): 649-656. DOI: https://doi.org/10.1097/00000421-198212000-00014

19. Hasenclever D, Diehl V, Armitage JO, et al. A prognostic score for advanced Hodgkin's disease. N Engl J Med Overseas Ed 1998; 339(21): 1506-1514. DOI: https://doi.org/10.1056/NEJM199811193392104

20. Charlson ME, Pompei $\mathrm{P}$, Ales KL, et al. A new method of classifying prognostic comorbidity in longitudinal studies: development and validation. J Chronic Dis 1987; 40(5): 373-383. DOI: https://doi.org/10.1016/0021-9681(87) 90171-8

21. Charlson M, Szatrowski TP, Peterson J, et al. Validation of a combined comorbidity index. J Clin Epidemiol 1994; 47(11): 1245-1251. DOI: https://doi.org/10.1016/0895-4356(94)90129-5

22. Quan $H$, Sundararajan V, Halfon $P$, et al. Coding algorithms for defining comorbidities in ICD-9-CM and ICD-10 administrative data. Med Care 2005; 43(11): 1130-1139. DOI: https://doi.org/10.1097/01.mlr.0000182534.19832. 83

23. Maringe $\mathrm{C}$, Fowler $\mathrm{H}$, Rachet $\mathrm{B}$, et al. Reproducibility, reliability and validity of population-based administrative health data for the assessment of cancer non-related comorbidities. PLoS One 2017; 12(3): e0172814. DOI: https:// doi.org/10.1371/journal.pone.0172814

24. Elliss-Brookes L, McPhail S, Ives A, et al. Routes to diagnosis for cancer - determining the patient journey using multiple routine data sets. Br J Cancer 2012; 107(8): 1220-1226. DOI: https://doi.org/10.1038/bjc.2012. 408

25. Kane E, Howell D, Smith A, et al. Emergency admission and survival from aggressive non-Hodgkin lymphoma: a report from the UK's population-based Haematological Malignancy Research Network. Eur J Cancer 2017; 78: 53-60. DOI: https://doi.org/10.1016/j.ejca.2017.03.013 
26. National Cancer Registration and Analysis Service. Routes to diagnosis: exploring emergency presentations. 2013; http://www.ncin.org.uk/publications/data_briefings/routes_to_diagnosis_exploring_emergency_presentations (accessed 20 Nov 2019).

27. Department for Communities and Local Government. The English Indices of Deprivation 2007. 2008; https:// webarchive.nationalarchives.gov.uk/20100411141238/http://www.communities.gov.uk/communities/ neighbourhoodrenewal/deprivation/deprivation07/ (accessed accessed 27 Nov 2019).

28. Estève J, Benhamou E, Croasdale $\mathrm{M}$, et al. Relative survival and the estimation of net survival: elements for further discussion. Stat Med 1990; 9(5): 529-538. DOI: https://doi.org/10.1002/sim.4780090506

29. Cancer Research UK Cancer Survival Group. UK Life Tables. 2017; http://csg.lshtm.ac.uk/tools-analysis/uk-lifetables/ (accessed 20 Nov 2019).

30. Cole SR, Hernán MA. Adjusted survival curves with inverse probability weights. Comput Methods Programs Biomed 2004; 75(1): 45-49. DOI: https://doi.org/10.1016/j.cmpb.2003.10.004

31. Radford J, Illidge T, Counsell N, et al. Results of a trial of PET-directed therapy for early-stage Hodgkin's lymphoma. N Engl J Med 2015; 372(17): 1598-1607. DOI: https://doi.org/10.1056/NEJMoa1408648

32. Johnson $\mathrm{P}$, Federico $\mathrm{M}$, Kirkwood $\mathrm{A}$, et al. Adapted treatment guided by interim PET-CT Scan in advanced Hodgkin's lymphoma. N Engl J Med 2016; 374(25): 2419-2429. DOI: https://doi.org/10.1056/NEJMoa1510093

33. Swerdlow S, Campo E, Harris N, et al WHO classification of tumours of haematopoietic and lymphoid tissues. Lyon: World Health Organization; 2017.

34. Allemani C, Matsuda T, Di Carlo V, et al. Global surveillance of trends in cancer survival 2000-14 (CONCORD-3): analysis of individual records for 37513025 patients diagnosed with one of 18 cancers from 322 population-based registries in 71 countries. Lancet 2018; 391(10125): 1023-1075. DOI: https://doi.org/10.1016/S0140-6736(17) 33326-3

35. Abel GA, Mendonca SC, McPhail S, et al. Emergency diagnosis of cancer and previous general practice consultations: insights from linked patient survey data. Br J Gen Pract 2017; 67(659): e377-e387. DOI: https://doi. org/10.3399/bjgp17X690869

36. National Cancer Registration and Analysis Services. Routes to diagnosis. 2010; http://www.ncin.org.uk/ publications/routes_to_diagnosis (accessed 26 Nov 2019).

37. Brenner H, Gondos A, Pulte D. Ongoing improvement in long-term survival of patients with Hodgkin disease at all ages and recent catch-up of older patients. Blood 2008; 111(6): 2977-2983. DOI: https://doi.org/10.1182/blood2007-10-115493

38. McPhail S, Elliss-Brookes L, Shelton J, et al. Emergency presentation of cancer and short-term mortality. Br J Cancer 2013; 109(8): 2027-2034. DOI: https://doi.org/10.1038/bjc.2013.569

39. Abel GA, Shelton J, Johnson S, et al. Cancer-specific variation in emergency presentation by sex, age and deprivation across 27 common and rarer cancers. Br J Cancer 2015; 112(S1): S129-. DOI: https://doi.org/10.1038/ bjc.2015.52

40. Björkholm M, Svedmyr E, Sjöberg J. How we treat elderly patients with Hodgkin lymphoma. Curr Opin Oncol 2011; 23(5): 421-. DOI: https://doi.org/10.1097/CCO.0b013e328348c6c1

41. Björkholm M, Weibull CE, Eloranta S, et al. Greater attention should be paid to developing therapies for elderly patients with Hodgkin lymphoma - a population-based study from Sweden. Eur J Haematol 2018; 101(1): 106-114. DOI: https://doi.org/10.1111/ejh.13090

42. Mitchell ED, Rubin G, Merriman L, et al. The role of primary care in cancer diagnosis via emergency presentation: qualitative synthesis of significant event reports. Br J Cancer 2015; 112(S1): S50-S56. DOI: https://doi.org/10.1038/ bjc. 2015.42

43. Lyratzopoulos G, Neal RD, Barbiere JM, et al. Variation in number of general practitioner consultations before hospital referral for cancer: findings from the 2010 National cancer patient experience survey in England. Lancet Oncol 2012; 13(4): 353-365. DOI: https://doi.org/10.1016/S1470-2045(12)70041-4

44. Mendonca SC, Abel GA, Saunders CL, et al. Pre-referral general practitioner consultations and subsequent experience of cancer care: evidence from the English cancer patient experience survey. Eur J Cancer Care 2016; 25(3): 478-490. DOI: https://doi.org/10.1111/ecc.12353

45. Howell DA, Hart RI, Smith AG, et al. Myeloma: patient accounts of their pathways to diagnosis. PLoS One 2018; 13(4): e0194788. DOI: https://doi.org/10.1371/journal.pone.0194788

46. Herbert A, Abel GA, Winters S, et al. Are inequalities in cancer diagnosis through emergency presentation narrowing, widening or remaining unchanged? Longitudinal analysis of English population-based data $2006-2013$. J Epidemiol Community Health 2019; 73(1): 3-10. DOI: https://doi.org/10.1136/jech-2017-210371 